\title{
Comparative sensitivities of various tests for diagnosing early Schistosoma mansoni infection in mice
}

\author{
Mahmoud Mohamed Bahgat ${ }^{1,3 *}$, Abduallh M. Ibrahim ${ }^{2}$, Amany Sayed Maghraby ${ }^{1,3}$, Maha Rizk ${ }^{3}$ and Rehab Abdel Megeed ${ }^{3}$ \\ ${ }^{1}$ Therapeutical Chemistry Department, Immunology and Infectious Diseases group, Center of Excellence for Advanced Sciences, the National \\ Research Center, Dokki, Cairo 12311, Egypt \\ ${ }^{2}$ Zoology Department, Faculty of Science, Ain Shams University, Cairo, Egypt \\ ${ }^{3}$ Therapeutical Chemistry Department, the National Research Center, Dokki, Cairo 12311, Egypt
}

\begin{abstract}
Objective: We compared the diagnostic values of cercarial antigen preparation, cercarial secretions, soluble worm antigen preparation and worm vomit prepared from the parasite Schistosoma mansoni.

Methods: Enzyme linked immunosorbant assay was used to detect IgG in plasma from Schistosoma mansoni infected mice. In parallel, specific primers for the parasite genome was used to detect S. mansoni DNA in plasma and urine from infected mice and hemolymph and tissues of infected Biomphalaria alexandrina snails by Polymerase chain reaction.

Results: The results showed that all the above diagnostic approaches enabled infection to be diagnosed as early as three days post mice exposure to parasite cercariae.

Conclusion: Cercarial secretions and worm vomit represent new useful economic crude antigens for preliminary detection of parasite active transmission or response to therapy in an endemic setting. Also, it was found that the detection of Schistosoma mansoni DNA in urine from infected mice was the most sensitive and specific (although expensive) method for infection diagnosis than all the others.
\end{abstract}

Keywords: Schistosoma mansoni; Cercarial secretion; Worm vomit; Soluble worm antigen preparation; Polymerase chain

\section{Introduction}

Diagnosis of schistosomiasis infection is conventionally carried out by Kato Katz technique [1-4] or serologic methods [5-6]. Using schistosome genome specific primers, allowed detection of the parasite DNA by polymerase chain reaction (PCR) in biological samples from infected subjects with superior specificity and sensitivity than the parasitological or the serological methods [7-14], as demonstrated by the capacity of the PCR to detect parasite DNA as early as 7 days post infection. While, the levels of the anti-worm $\operatorname{IgG}$ remained high at 23 weeks post-treatment, the PCR results turned negative at week 10 posttreatment [14].

Herein we compared the diagnostic values of several crude antigens prepared from the cercarial and the adult worm stages of the parasite $S$. mansoni to detect IgG in sera from infected mice with the same parasite kinetically at regular time intervals post infection. Of these antigens cercarial secretaions (CS) and worm vomit (WoV) were previously evaluated for their diagnostic value to detect IgG in sera from infected humans [15]. In parallel, and in a trial to improve the sensitivity and specificity of the diagnosis, a PCR based on specific primers for the parasite genome was evaluated for its capacity to detect infection in plasma and urine from infected mice.

\section{Materials and Methods}

\section{Infected mice groups and samples collection}

Fifty Swiss albino mice were divided into 10 groups each of 5 and each mouse received 100 cercariae by tail immersion. During the acute phase (the initial 30 days post infection), every 3 days starting from day 3 post infection urine was pooled from each group and individual blood samples were collected on EDTA. Plasma was separated and stored at $-20^{\circ} \mathrm{C}$ till being used. Plasma and urine were collected from 20 uninfected mice to be used as negative controls. Tissues and hemolymph were collected from infected B. alexandirina for detection of S. mansoni DNA by PCR. Tissues from uninfected snails were examined as means of negative controls.

\section{Molecular detection}

gDNA extraction: DNA was extracted from different stages of $S$. mansoni (worm, cercaria and schistosomula) and tissue from infected $B$. alexandrina snails using lysis buffer containing $2 \%$ hexadecyltrimethylammonium bromide (CTAB) with the method reported by AbdelHamid et al. [16]. DNA was also extracted from plasma and urine of infected mice using wizard gDNA purification kit (Promega).

Bioinformatics analysis of the used primers: The used forward (ACAGTGCGCGCGTCGTAAGC) and reverse (GAGATCAAGTGTGACAGTTTTGC) primers were previously reported to specifically amplify $S$. mansoni gDNA [10]. The sequences of both primers were compared by multiple alignments against the online available sequences in the GenBank (http://www.ncbi.nlm.nih.gov/) using the basic nucleotide BLAST analysis.

Amplification of gDNA: PCR reactions were carried out in a

*Corresponding author: Mahmoud Mohamed Bahgat, Department of Infection Genetics, The Helmholtz Center for Infection Research, Inhoffenstrasse 7, D-38124 Braunschweig, Germany, Fax: 00202-33370931/+49-0531-6181-1199; E-mail: mbahgatriad@yahoo.com

Received April 05, 2011; Accepted June 15, 2011; Published June 28, 2011

Citation: Bahgat MM, Ibrahim AM, Maghraby AS, Rizk M, Megeed RA (2011) Comparative sensitivities of various tests for diagnosing early Schistosoma mansoni infection in mice. J Bacteriol Parasitol 2:116. doi:10.4172/2155-9597.1000116

Copyright: @ 2011 Bahgat MM, et al. This is an open-access article distributed under the terms of the Creative Commons Attribution License, which permits unrestricted use, distribution, and reproduction in any medium, provided the original author and source are credited. 
Citation: Bahgat MM, Ibrahim AM, Maghraby AS, Rizk M, Megeed RA (2011) Comparative sensitivities of various tests for diagnosing early Schistosoma mansoni infection in mice. J Bacteriol Parasitol 2:116. doi:10.4172/2155-9597.1000116

Page 2 of 5

final volume of $50 \mu \mathrm{l}$. The amplification program included an initial denaturation at $94 \mathrm{oC}$ for $5 \mathrm{~min}$ followed by 35 cycles; each consisted of denaturation at $94 \mathrm{oC}$ for $45 \mathrm{sec}$, annealing at $58 \mathrm{oC}$ for $45 \mathrm{sec}$ and extension at $72 \mathrm{oC}$ for $60 \mathrm{sec}$. The program included a final extension step at $72 \mathrm{oC}$ for $10 \mathrm{~min}$. PCR products were resolved by electrophoresis on $1.5 \%$ agarose gel containing ethidium bromide [17] parallel to $100 \mathrm{bp}$ DNA ladder (promega, Germany). The amplified S. mansoni fragment was visualized using an UV-transilluminator and photographed using a gel documentation system.

\section{Serological detection}

Preparation of antigens: CAP AND SWAP WERE PREPARED ACCORDING TO THE METHOD DESCRIBED BY JASSIM ET AL [18]. CS AND WOV WERE PREPARED ACCORDING TO THE METHOD OF BAHGAT ET AL $[15,19]$.

Detection of IgG levels in plasma from infected mice against CAP, SWAP, CS and WoV [20]: ELISA plates were coated overnight at room temperature with $50 \mu \mathrm{l} /$ well of CAP $(62.5 \mu \mathrm{g} / \mathrm{ml})$, CS $(20 \mu \mathrm{g} /$ $\mathrm{ml})$, SWAP $(250 \mu \mathrm{g} / \mathrm{ml})$ and WoV $(200 \mu \mathrm{g} / \mathrm{lm})$ in coating buffer. Plates were washed 3 times with PBS-0.05\% T20 and blocked against non specific binding with $100 \mu \mathrm{l} /$ well of PBS- $0.05 \%$ T-5\% fetal calf sera (FCS) for $2 \mathrm{~h}$ at $37^{\circ} \mathrm{C}$. After three washes, individual plasma samples were diluted $1 / 100$ in the blocking buffer and $50 \mu$ of each were applied to individual wells. Plates were incubated at $37^{\circ} \mathrm{C}$ for $2 \mathrm{~h}$. After washing, diluted peroxidase labeled anti-mouse IgG (1/500) in PBS-0.05\% T-5\% FCS was then applied $50 \mu \mathrm{l} /$ well and plates were incubated $2 \mathrm{~h}$ at $37^{\circ} \mathrm{C}$ followed by 3 washes. For visualizing the antigen antibody binding, plates were dried and the peroxidase specific colorimetric substrate O-phenylenediamine dihydrochloride (Sigma, St. Louis, Mo, USA) appropriately diluted in the substrate buffer in presence of $\mathrm{H}_{2} \mathrm{O}_{2}$ was applied $50 \mu \mathrm{l} /$ well.
Statistical analysis: All obtained data were analyzed using the Student's t-test application of the Graph Pad InStat Soft ware.

\section{Results}

Detection of IgG levels by ELISA in plasma from $S$. mansoni infected mice: As presented in Table 1, using CAP coated plates, IgG levels in infected mice plasma (IMP) showed no significant increases at 3, 6 and 15 days post infection, (1.4, 2.2 and 1.9, folds respectively) compared with IgG levels in uninfected (UIMP), while at 9, 12, 18, 21, 24, 27 and 30 days IgG levels in IMS were significantly higher $(\mathrm{P}<$ 0.05) than in UIMP. Using CS coated plates, IgG levels in IMP were significantly higher $(\mathrm{P}<0.05)$ than in UIMP in all intervals except at 15 day post infection, IgG levels showed no significant increases (1.96 fold). On the other hand, using SWAP coated plates, at all time intervals IgG levels in IMS were significantly higher $(\mathrm{P}<0.05)$ than in UIMP while, Using WoV coated plates, IgG levels in IMP were significantly higher than in UIMP at all intervals except at 21 day, where, IgG levels in IMP showed no significant increases (1.59 fold).

Mean percentage IgG reactivities in IMP against CAP, CS, SWAP and WoV over 30 days post infection: IgG reactivities in IMP against the used antigens were considered positive if they exceeded the cut off values for each antigen that were calculated as the means of the UIMP IgG reactivities + 2X Standard deviation (S.D.) and were 0.27, 0.32, 0.39 and 0.52 for CS, SWAP, CAP and WoV respectively (Table 1).

Molecular analysis: Using the basic nucleotide blast application, results showed $100 \%$ homology to the highlighted termini of the used primers suggesting their exact annealing positions on the parasite template that yields amplification products of molecular size $338 \mathrm{bp}$ (Figure 1). Such analysis confirmed the specificity of the used primers

\begin{tabular}{|c|c|c|c|c|c|c|c|c|c|c|c|}
\hline \multicolumn{12}{|c|}{ IgG reactivity against CAP at different time intervals of infection } \\
\hline & C & 3D & 6D & 9D & 12D & 15D & 18D & 21D & 24D & 27D & 30D \\
\hline Mean & 0.22 & 0.31 & 0.49 & 0.53 & 0.47 & 0.42 & 0.64 & 0.622 & 0.602 & 0.538 & 0.75 \\
\hline SD & 0.08 & 0.02 & 0.26 & 0.09 & 0.01 & 0.21 & 0.22 & 0.12 & 0.21 & 0.07 & 0.11 \\
\hline P- value & & $0.08 \mathrm{NS}$ & $0.09 \mathrm{NS}$ & $0.001^{*}$ & $0.006^{*}$ & $0.12 \mathrm{NS}$ & $0.01^{*}$ & $0.001^{*}$ & $0.013^{*}$ & $0.0005^{*}$ & $0.0001^{*}$ \\
\hline Cut off & 0.39 & $0 \%$ & $60 \%$ & $100 \%$ & $80 \%$ & $20 \%$ & $80 \%$ & $100 \%$ & $80 \%$ & $100 \%$ & $100 \%$ \\
\hline \multicolumn{12}{|c|}{ IgG reactivity against $\mathrm{CS}$ at different time intervals of infection } \\
\hline & C & 3D & $6 D$ & 9D & 12D & 15D & 18D & 21D & 24D & 27D & 30D \\
\hline Mean & 0.25 & 0.37 & 0.6 & 0.46 & 0.36 & 0.49 & 0.61 & 0.65 & 0.679 & 0.638 & 0.79 \\
\hline SD & 0.01 & 0.1 & 0.02 & 0.16 & 0.07 & 0.28 & 0.25 & 0.21 & 0.14 & 0.1 & 0.27 \\
\hline P-value & & $0.03^{*}$ & $0.003^{*}$ & $0.01^{*}$ & $0.007^{*}$ & $0.07 \mathrm{NS}$ & $0.007^{*}$ & $0.001^{*}$ & $0.001^{*}$ & $0.001^{*}$ & $0.001^{*}$ \\
\hline Cut off & 0.27 & $80 \%$ & $100 \%$ & $100 \%$ & $80 \%$ & $100 \%$ & $100 \%$ & $100 \%$ & $100 \%$ & $100 \%$ & $100 \%$ \\
\hline \multicolumn{12}{|c|}{ IgG reactivity against SWAP at different time intervals of infection } \\
\hline & C & 3D & 6D & 9D & 12D & 15D & 18D & 21D & 24D & 27D & 30D \\
\hline Mean & 0.24 & 0.68 & 0.69 & 0.43 & 0.47 & 0.6 & 0.51 & 0.537 & 0.747 & 0.57 & 0.55 \\
\hline SD & 0.06 & 0.17 & 0.22 & 0.05 & 0.1 & 0.19 & 0.08 & 0.15 & 0.09 & 0.13 & 0.11 \\
\hline P-value & & $0.0007^{*}$ & $0.002^{*}$ & $0.001^{*}$ & $0.003^{*}$ & $0.0005^{*}$ & $0.0006^{*}$ & $0.005^{*}$ & $0.0001^{*}$ & $0.001^{*}$ & $0.0007^{*}$ \\
\hline Cut off & 0.32 & $100 \%$ & $100 \%$ & $100 \%$ & $100 \%$ & $80 \%$ & $100 \%$ & $100 \%$ & $100 \%$ & $100 \%$ & $100 \%$ \\
\hline \multicolumn{12}{|c|}{ IgG reactivity against WoV at different time intervals of infection } \\
\hline & C & 3D & 6D & 9D & 12D & 15D & 18D & 21D & 24D & 27D & 30D \\
\hline Mean & 0.32 & 0.53 & 0.6 & 0.64 & 0.49 & 0.57 & 0.54 & 0.51 & 0.83 & $0.81^{`}$ & 0.87 \\
\hline SD & 0.1 & 0.12 & 0.12 & 0.1 & 0.07 & 0.14 & 0.12 & 0.2 & 0.09 & 0.13 & 0.14 \\
\hline P-value & & $0.01^{*}$ & $0.006^{*}$ & $0.002^{*}$ & $0.01^{*}$ & $0.01^{*}$ & $0.01^{*}$ & $0.1 \mathrm{CNS}$ & $0.0001^{*}$ & $0.0002^{*}$ & $0.0001^{*}$ \\
\hline Cut off & 0.52 & $40 \%$ & $80 \%$ & $80 \%$ & $20 \%$ & $80 \%$ & $60 \%$ & $60 \%$ & $100 \%$ & $100 \%$ & $100 \% \mathrm{t}$ \\
\hline
\end{tabular}

*Significant value $\mathrm{P}<0.05$

Cut off: 2 mean \pm SD for control

N.S.: non significant

$(P<0.05)$ 
Citation: Bahgat MM, Ibrahim AM, Maghraby AS, Rizk M, Megeed RA (2011) Comparative sensitivities of various tests for diagnosing early Schistosoma mansoni infection in mice. J Bacteriol Parasitol 2:116. doi:10.4172/2155-9597.1000116

to $S$. mansoni genome as they did not show any extent of homology to other existing human parasites in Egypt (Figure 1).

PCR amplification of the target sequence on the gDNA from different $S$. mansoni stages using the species specific primers: Specific amplification products from gDNA of adult $S$. mansoni worms, schistosomula, cercariae (lanes 4, $5 \& 6$ ), infected snail's tissues (lanes 7 , 8 ) as well as hemolymph (lanes 9,10 ) were visualized at 338 bp (Figure 2 ) that were not detected in the negative control lanes $(2,3)$.

PCR amplification of the target $S$. mansoni sequence from gDNA extracted from IMP and mice urine samples: DNA extracted from IMP then subjected to PCR amplification demonstrated $338 \mathrm{bp}$ amplification products (Figure 3 ) as early as 3 days post infection that were persistently detectable over 30 days post infection. On the other hand, all gDNA preparations from collected mice urine samples at the $3 \mathrm{rd}$, 9th, 12th, 15th, 18th, 21st and 30th days post infection were consistently PCR positive for the $338 \mathrm{bp}$ fragment. At the 6th, 24th and 27th days post infection PCR was not carried out due to the insufficient collected urine volume (less than $100 \mu \mathrm{l}$ ) for gDNA extraction (Figure 4).

\section{Discussion}

A diagnostic tool capable of detecting Schistosoma infections in its acute phase would be of great value permitting early treatment that could prevent the pathology associated with chronic infections.

In the present work, the recorded high IgG levels against CAP

\section{GAGATCAAG TGTGACAG TTTTGCTCTGAGCT ACCCTGGA TCGGGTTG T}

TTGTGAA TGCAGCCCAAAGTGGGTGG TAAACTCCA TCA GGCTAAATACTT

\begin{abstract}
ACACGAGTCCG ATAGCAAACAAGT ACCGGGAAAGTTGAAAGT ACTTTG
\end{abstract}
AAGAGAGAGT AAACAGTGCGTGAACCGCT AAAGGT AAACGGGTGGAG T

TGAACTGCAAGCTCTGGGAA TTCAGCTGA TGAGTGTGA TTTGTACTTGGG

CATACTGGCCGCCTTCAGTGTCTGTTT AACCGCAGGTGCCTTCCTTTTTGG

TGGGTATGTGTGAA TCGTTTGCTT ACGACGCGCGCACTGT

Figure 1: Deduce target $S$. mansoni sequence for the PCR amplification using the previously published primers (Sandoval, et al., 2006 a \& b). Sequences of both the forward and reverse primers were compared with the published schistosome sequences on the GenBank (http://www.ncbi.nlm.nih.gov) using the basic nucleotide blast application for and results showed $100 \%$ homology to the highlighted termini of the below mentioned previously S. mansoni sequence suggesting their exact annealing positions on the parasite template.

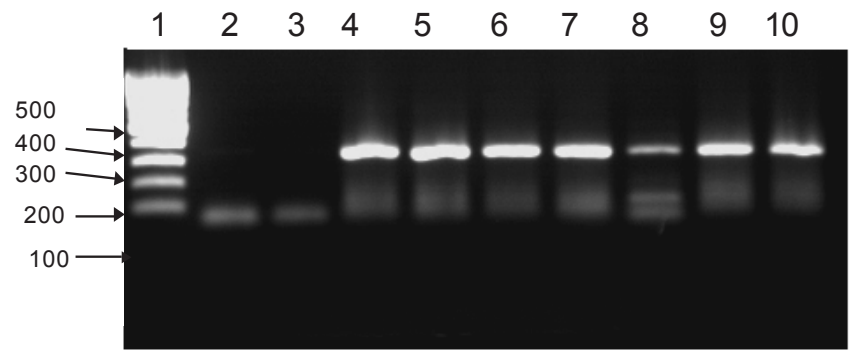

Figure 2: Electrophoresis of amplification products from genomic DNA of various parasite developmental stages. PCR products of adult $S$. mansoni worms, schistosomula, cercariae (lanes $4,5 \& 6$ ), infected $B$. alexandrina snail tissues (lanes $7 \& 8$ ) as well as hemolymph (lanes $9 \& 10$ ) were subjected for electrophoresis on $1.5 \%$ agarose gel containing ethidium bromide parallel to 100 bp DNA ladder (lane 1) and products from a negative control of PCR reaction (lanes 2 \& 3) where no DNA was added in the master mix were included on the same gel. Specific amplification products at 338 bp were visualized in all developmental stages that were not evidenced in the negative control lanes.

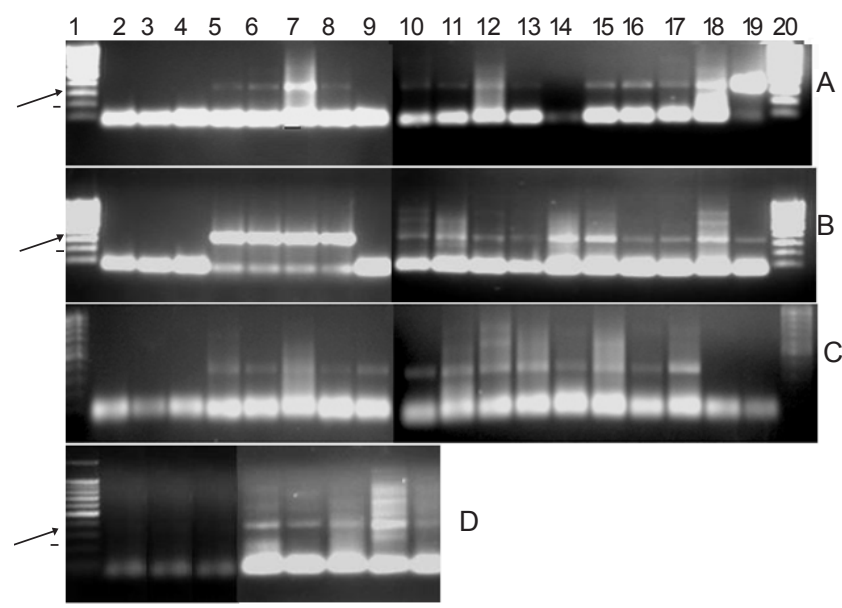

Figure 3: Electrophoresis of amplification products from genomic DNA of infected mice plasma (IMP) at different time points post infection. At 3, 6 and 12 days post infection 4 out of the tested 5 IMP samples at each of the 3 time points were PCR positive (A; lanes $5-8$ \& $10-13, B$; $6-9$ ) while, 1 sample at each of the 3 time points was PCR negative (A; lanes 9\&14, B lane 5). At 9 $15,18,21,24$ and 30 days post infection all the 5 tested IMP samples at each of such time points were uniformly PCR positive (A; lanes 15-19, B; 10-14\&1519, C; 5-9\&10-14, D; 57-9). At 27 days post infection, 3 samples were PCR positive (C; lanes 15-17), while, two were negative (C; lanes 17\&18). That were subjected for electrophoresis on $1.5 \%$ agarose gel containing ethidium bromide parallel to 100 bp DNA ladder (lane 1\&20), products from a negative control of PCR reaction (lane 2), and products from control of UIMP (3 \&4), where 338 bp specific amplification products were visualized in all positive samples that were not evidenced in the negative control lane.

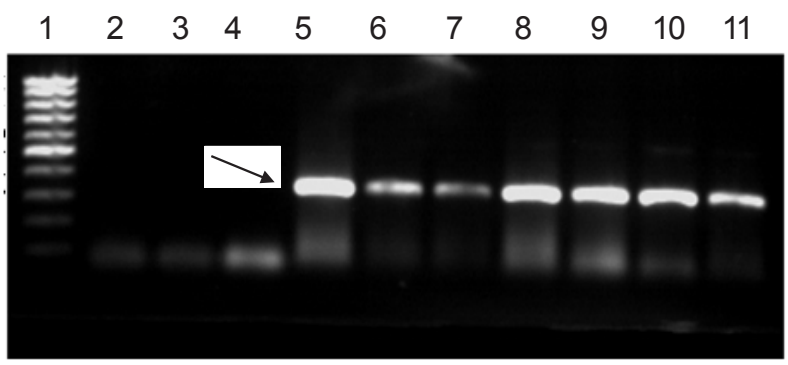

Figure 4: Electrophoresis of amplification products from genomic DNA of infected mice urine at different time points post infection. At 3rd, 9th 12th, 15th, 18th, 21st and 30th $(5,6,7,8,9,10$ \& 11espectively) were subjected for electrophoresis on $1.5 \%$ agarose gel containing ethidium bromide parallel to $100 \mathrm{bp}$ DNA ladder (lane 1), products from a negative control of PCR reaction (lane 2) and control of non infected urine ( 3 \& 4) PCR reaction where 338 bp specific amplification products were visualized at all time points that were not evidenced in the both control and negative control lanes.

at different time intervals post infection agrees with the previously reported high IgG levels in sera of $S$. mansoni infected patients predominantly against the abundant carbohydrate epitopes in the cercarial antigens [21], an increase in IgG at all intervals post infection against SWAP agrees with the previously reported high sensitivity of such antigenic preparation to detect IgG in sera from patients infected with S. mansoni [22].

The antigenicity of the SWAP might be attributed to the carbohydrate epitopes on glycoconjugates either presented or excreted by adult worms and elicit strong humoral immune response or the $\mathrm{O}$-glycans of the major gut-associated CAA [23]. 
The present results clearly demonstrated that CS sensitivity to detect IgG in plasma of $S$. mansoni infected mice was generally higher than that of WoV which contrasted the previous study that compared the sensitivity of the two antigens to detect IgG in sera of infected humans residing in an endemic area in Burkina Faso [15]. Furthermore, antibodies against certain antigens present in the worm intestine, may take longer time to disappear [24].

The superior sensitivity of the CS to detect IgG agrees with the previous report [25] that described cercarial elastase (CE) purified from S. mansoni larval secretions as marker for human exposure to $S$. mansoni cercariae and not necessarily infection that was later contradicted and clearly demonstrated that natural infection induces antibodies to cercarial antigens present in the CS but not to the CE [26].

Using previously reported primers [10] in PCR, allowed amplification of specific fragments with the expected molecular weight (338 bp) from gDNA extracted from growing sporocysts within infected B. alexandrina snails, cercaria, schistosomula and adult S. mansoni worms that confirmed the sensitivity of the used primers. Such results match with those reported by [10] to establish PCR allowing specific amplification of a genus-specific product from the $28 \mathrm{~S}$ ribosomal DNA subunit of Schistosoma spp.

The most impressive finding in the present work was that parasite DNA started to be detectable in IMP as early as 3 days post infection using PCR and continued to be detectable for 30 days, reflecting the superior sensitivity of such diagnostic tool for detecting infection at a very early stage.

Six out of ten time points reached $100 \%$ sensitivity, three time points declared $80 \%$ and only one time point showed $60 \%$ sensitivity. These results match with those from previous study [8] in which amplification was achieved with minimum amounts of $S$. mansoni egg template DNA (1fg) in human serum and feces. Also, 97\% of PCR sensitivity to detect infection, demonstrating that single PCR survey detected more cases of S. mansoni infections than three Kato-Katz stool examinations [9].

The sensitivity of ELISA against the four used antigens reached $100 \%$ compared to $60 \%$ PCR sensitivity at day 27 . However, this indicates that false-positive results might have been observed due to cross-reactivity with other helminth infections but this can be ruled, since the DNA from these worms is not amplified by the S. mansonispecific primers $[10,12]$.

Due to the facts that the collection of blood is invasive and collection of stool may be difficult in some populations, thus hampering diagnostic procedures, therefore that PCR was applied on gDNA extracted from pooled mice urine at different time intervals post infection.

In the present work, parasitic DNA was also detectable by PCR in extracted gDNA from pooled mice urine as early as three days post infection that remained detectable over 30 days reflecting the extremely high sensitivity of such detection. This agrees with a previous study to detect $S$. mansoni infection in mice urine by PCR after 7 days post infection [11]. The obtained $100 \%$ sensitivity of detecting S. mansoni DNA by PCR in urine in the current study was consistent with a previous study [10].

In the present study, $80 \mu$ l of plasma and $400 \mu$ l of urine were sufficient to extract gDNA for detecting S. mansoni infection by PCR. However, three $\mathrm{ml}$ of human urine sample for parasite detection by PCR [10]. Furthermore, S. mansoni infection could be detected from only $2 \mu l$ of infected mice sera by direct PCR without DNA purification [10].
The general superiority of CS and WoV as new antigenic preparations to detect infection in IMP at different time points post infection clearly demonstrates a more economic and easy to prepare antigen that can be used to detect infection in poor endemic settings, yet, the specificity of detection remains as an opened question. However, PCR seems to be the best in terms of both sensitivity and specificity, yet, the decision on using it will always depend on the cost.

It could be concluded that in the acute infection phase, PCR detection of $S$. mansoni infection in urine samples is non invasive, very sensitive and specific diagnostic method than immunodiagnosis or even PCR detection of infection in sera

\section{Acknowledgements}

This work was supported by several awarded grants from the National Research Center of Egypt. The authors acknowledge Prof. Abdel Hamid Zaki for his moral support, Hanaa M. Gaber for her technical help in the serology part, Kareem Awad for giving CS, WoV antigens and Rabeh El Shishiny for his help in dissecting mice.

\section{References}

1. Katz N, Chaves A, Pellegrino J (1972) A simple device for quantitative stool thick-smear technique in schistosomiasis mansoni. Rev Inst Med Trop Sao Paulo 14: 397-400

2. Jurberg $A D$, Oliveira $A A$, Lenzi $H L$, Coelho $P M(2008) A$ new miracidia hatching device for diagnosing schistosomiasis. Mem Inst Oswaldo Cruz 103: 112-114.

3. Allam AF, Kader O, Zaki A, Shehab AY, Farag HF (2009) Assessing the marginal error in diagnosis and cure of Schistosoma mansoni in areas of low endemicity using Percoll and PCR techniques. Trop Med Inter Health 14: 316-321.

4. Tweyongyere R, Mawa PA, Emojong NO, Mpairwe H, Jones FM, et al. (2009) Effect of praziquantel treatment of Schistosoma mansoni during pregnancy on intensity of infection and antibody responses to schistosome antigens: results of a randomised, placebo-controlled trial. BMC Infectious Disease 18: 32.

5. Corstjens PL, van Lieshout L, Zuiderwijk M, Kornelis D, Tanke HJ, et al. (2008) Up-converting phosphor technology-based lateral flow assay for detection of Schistosoma circulating anodic antigen in serum. J Clinic Microbial 46: 171-176.

6. Midzi N, Butterworth AE, Mduluza T, Munyati S, Deelder AM, et al. (2009) Use of circulating cathodic antigen strips for the diagnosis of urinary schistosomiasis. Trans Roy Soc Trop Med Hyg 103: 45-51.

7. Rabello A, Pontes LA, Dias-Neto E (2002) Recent advances in the diagnosis of Schistosoma infection: the detection of parasite DNA. Mem Inst Oswaldo Cruz 97: 171-172.

8. Pontes LA, Dias-Neto E, Rabello A (2002) Detection by polymerase chain reaction of Schistosoma mansoni DNA in human serum and faeces. Am J Trop Med Hyg 66: 157-162.

9. Pontes LA, Oliveira MC, Katz N, Dias-Neto E, Rabello A (2003) A comparison of polymerase chain reaction and the Kato-Katz technique for diagnosing infection with Schistosoma mansoni. Am J Trop Med Hyg 68: 52-56.

10. Sandoval N, Siles-Lucas M, Pérez-Arellano JL, Carranza C, Puente S, et al. (2006a) A new PCR-based approach for the specific amplification of DNA from different Schistosoma species applicable to human urine samples. Parasitology 133: $581-587$

11. Sandoval N, Siles-Lucas M, Lopez Aban J, Pérez-Arellano JL, Gárate T, et al. (2006b) Schistosoma mansoni: A diagnostic approach to detect acute Schistosomiasis infection in a murine model by PCR. Exp Parasitol 114: 84-88.

12. Suzuki T, Osada Y, Kumagai T, Hamada A, Okuzawa E, et al. (2006) Early detection of Schistosoma mansoni infection by touchdown PCR in a mouse model. Parasitol Int 55: 213-218.

13. Lier T, Johansen MV, Hjelmevoll SO, Vennervald BJ, Simonsen GS (2008) Real-time PCR for detection of low intensity Schistosoma japonicum infections in a pig model. Acta Tropica 105: 74-80.

14. Xia CM, Rong R, Lu ZX, Shi CJ, Xu J, et al. (2009) Schistosoma japonicum: a PCR assay for the early detection and evaluation of treatment in a rabbit model. Exp Parasitol 121:175-179. 
Citation: Bahgat MM, Ibrahim AM, Maghraby AS, Rizk M, Megeed RA (2011) Comparative sensitivities of various tests for diagnosing early Schistosoma mansoni infection in mice. J Bacteriol Parasitol 2:116. doi:10.4172/2155-9597.1000116

Page 5 of 5

15. Bahgat M, Sorgho H, Ouédraogo JB, Poda JN, Sawadogo L, et al. (2006) Enzyme-linked immunosorbent assay with worm vomit and cercarial secretions of Schistosoma mansoni to detect infections in an endemic focus of Burkina Faso J Helminthol 80: 19-23.

16. Abdel-Hamid AH, de Molfetta JB, Fernandez V, Rodrigues V (1999) Genetic variation between susceptible and non-susceptible snails to Schistosoma infection using random amplified polymorphic DNA analysis (RAPDs). Rev Inst Med Trop Sao Paulo 41: 291-295.

17. Helling RB, Goodman HM, Boyer HW (1974) Analysis of R. EcoRI fragments of DNA from lambdoid bacteriophages and other viruses by agarose-gel electrophoresis. J Virol 14: 1235-1238.

18. Jassim A, Catty D, Hassan K (1987) Antibody isotypes in human schistosoma mansoni in Sudan. Parasite Immunol 9: 627-650.

19. Bahgat M, Ruppel A (2002) Biochemical comparison of the serine protease (elastase) activities in cercarial secretions from Trichobilharzia ocellata and Schistosoma mansoni. Parasitol Research 88: 495-500.

20. Maghraby A, Bahgat M (2004) Immunostimulatory effect of coumarin derivatives before and after infection of mice with the parasite Schistosoma mansoni. Arz Forschung 54: 545-550.

21. Nyame AK, Lewis FA, Doughty BL, Correa-Oliveira R, Cummings RD (2003)
Immunity to schistosomiasis: glycans are potential antigenic targets for immune intervention. Exp Parasitol 104: 1-13.

22. Sorgho H, Bahgat M, Poda JN, Song W, Kirsten C, et al. (2005) Serodiagnosis of Schistosoma mansoni infections in an endemic area of Burkina Faso: performance of several immunological tests with different parasite antigens. Acta Tropica 93:169-180.

23. Van Dam GJ, Bergwerff AA, Thomas-Oates JE, Rotmans JP, Kamerling JP, et al. (1994) The immunologically reactive O-linked polysaccharide chains derived from circulating cathodic antigen isolated from the human blood fluke Schistosoma mansoni have Lewis-X as a repeating unit. Europ J Biochem 225: 467-482.

24. Planchart S, Incani RN, Cesari IM (2007) Preliminary characterization of an adult worm "vomit" preparation of Schistosoma mansoni and its potential use as antigen for diagnosis. Parasitol Research 101: 301-309.

25. Ramzy RM, Faris R, Bahgat M, Helmy H, Franklin C, et al. (1997) Evaluation of a stage-specific proteolytic enzyme of Schistosoma mansoni as a marker of exposure. Am J Trop Med Hyg 56: 668-673.

26. Bahgat M, Francklow K, Doenhoff MJ, Li YL, Ramzy RM, et al. (2001) Infection induces antibodies against the cercarial secretions, but not against the cercarial elastases of Schistosoma mansoni, Schistosoma haematobium, Schistosoma japonicum and Trichobilharzia ocellata. Parasite. Immunol. 23: 557-565. 